\title{
Prevalence of HIV Infection among Trauma Patients Admitted to Bugando Medical Centre, Mwanza, Tanzania and its Influence on Outcome
}

\author{
VIRGNIA MAYALA, STEPHEN E. MSHANA ${ }^{1 *}$, PHILLIPO L. CHALYA, RAMASH M. DASS and \\ SAMUEL E. KALLUVYA \\ Weill Bugando University Collage of Health Sciences, P.O. Box 1464, Mwanza, Tanzania
}

\begin{abstract}
HIV infection, a major health problem worldwide, has been reported to be prevalent in trauma patients, thus presents an occupational hazard to health care workers who care for these patients. The purpose of this study was to establish the prevalence of HIV among trauma patients in our setting and to compare the outcome of these patients who are HIV positive with those who are HIV negative. This was a descriptive cross sectional study involving trauma patients aged 11 years and above, admitted to the surgical wards of Bugando Medical Centre in Mwanza, Tanzania over a six-month period from October 2008 to March 2009. All eligible patients were consecutively enrolled in the study. Data were collected using a pre-tested, coded questionnaire and analyzed using SPSS computer software. A total of 250 trauma patients were recruited and studied. The mean age of the study population was $36.37 \pm 15.35$. Males accounted for the majority $(N=202 ; 80.8 \%)$ of the study population. The prevalence of HIV among trauma patients was 11.6\%. Among the HIV positive patients, $26(89.7 \%)$ were males and majority aged 31-40 years. Seventy two percent of HIV positive patients had CD4+ count of $\geq 200$ cells/ $\mu$ l. Overall length of hospital stays (LOS) ranged from 1 - 90 days with mean of 19.11 \pm 15.84 days. Using multivariate logistic regression, injury severity score $(I S S)(P=0.0026)$, revised trauma scores $(R T S)(P=0.002)$, HIV seropositivity $(P=0.0012)$ and CD4 + count $(P=0.001)$ were significantly found to be associated with increased LOS. Mortality rate was $10.8 \%$ and was significantly associated with; the body region injured $(P<0.05), \operatorname{ISS}(P=0.026), \operatorname{RTS}(P=0.001), \operatorname{PTS}(P=0.01), H I V$ positivity $(P=0.0001)$ and $C D 4+$ count $(P=0.035)$. HIV is prevalent among trauma patients in our setting. A substantial risk of exposure to HIV exists in health workers who care for these patients. Thus, all trauma health care workers in this region need to practice universal barrier precautions in order to reduce the risk of exposure to HIV infection. HIV positive patients with $C D 4+$ count $\geq 200$ cells/ $\mu$ l have similar prognosis as HIV negative patients and therefore should be treated the same way.
\end{abstract}

Key words: HIV prevalence, trauma patients, outcome, hospital, Tanzania

\section{Introduction}

Trauma continues to be an enormous public health problem worldwide and it is associated with high morbidity and mortality both in developed and developing countries (WHO, 1999). Trauma is reported to be the leading cause of death among people between 1 and 44 years of age (Dee, 1999). In Bugando Medical Centre in Mwanza, Tanzania, trauma is the single commonest indication for admission reported in the surgical wards (BMC Medical Record Database, 2006-2007).

The management of trauma patients poses a challenge to trauma health care workers in developing countries where resources for caring are limited. The risk of HIV transmission has been reported to

\footnotetext{
* Corresponding author: Dr Stephen Mshana; mshana72@yahoo. com
}

be high in health care workers who care for trauma patients due to frequent contact with blood and body fluids during the resuscitation of HIV-infected patients (Risi et al., 1989). The risk of exposure to HIV in the management of trauma depends on the underlying prevalence of infection in the population. In developing nations, the population prevalence of $\mathrm{HIV}$ in some groups can be as high as 30\% (Risi et al., 1989; Hammond et al., 1990). In trauma population, the prevalence of HIV seropositivity have been reported to run as high as $19 \%$ and thus presents an occupational hazard to health care workers who care for these patients (Hammond et al., 1990). Studies have shown that the prevalence of HIV infection among trauma patients reflects that in the general population but is generally higher (Baraff et al., 1989; Kelen et al., 1989). Trauma health care workers will encounter patients infected 
with HIV in geographic areas where prevalence is high or in areas where intravenous drug abuse use, high-risk sexual behaviors and penetrating trauma are more common (Kelen et al, 1989). Studies of patients with penetrating trauma in the USA have shown a higher prevalence of HIV infection than the general population (Chambers \& Lord, 2001).

Universal precautions have been reported to reduce the rate of HIV transmission; however, studies have indicated poor compliance to universal precautions in developing countries where resources for caring of these patients are limited (Courington et al, 1991; Xeroulis et al, 2005). Thus, the risk of exposure to HIV infection among trauma health care workers in our setting is high.

The current seroprevalence of HIV in trauma population in our setting is unknown. Establishing the seroprevalence of HIV in trauma patients is vital for education and post-exposure prophylaxis. The objective of this study was to establish the prevalence of HIV among trauma patients admitted at Bugando Medical Centre in Mwanza, Tanzania and to compare the outcome of patients who are HIV positive with those who are HIV negative.

\section{Materials and Methods}

\section{Patients}

This cross-sectional study was conducted in the surgical wards of Bugando Medical Centre over a six-month period from October 2008 to March 2009. The study population included all trauma patients aged $\geq 11$ years and who consented for the study. Unconscious patients without next of kin to consent were excluded from the study. Patients with obvious co-morbid conditions and those who were still in the wards at the end of study period were also excluded from the study. Recruitment of patients was done at the emergency department and in the surgical wards after primary and secondary surveys done by the admitting surgical team. After informed consent to participate in the study and to be tested for HIV, all patients who met the inclusion criteria were consecutively enrolled into the study. When personal consent was not possible due to the nature of the injuries or age below 18 years, consent was sought from the family. Patients were assured that the result would not affect their management and were given the choice to know their results.

Under aseptic technique, $3 \mathrm{mls}$ of blood was obtained from the patient by venopuncture from one of the following sites: the antecubital fossa, dorsum of the hand, volar aspect of the forearm, or femoral vein. The sample was put in a container containing EDTA and used to carry out HIV serology test and CD4+ cell count. HIV serology test was performed using the Tanzania HIV Rapid Test Algorithm (Lyamuya et al, 2009). CD4+ cell count was performed using FACS Count or FACSCALIBUR (BD Biosciences USA), to all HIV positive patients to determine the degree of immunosuppression. Imaging studies like $\mathrm{x}$-rays, ultrasound and Computer Tomography were also taken depending on the type of injury.

All recruited patients were managed according to advanced trauma life support (ATLS). In case of burn injuries, associated injuries were managed appropriately according to the type of injury. The authors ensured that the study patients were receiving the appropriate treatment and supportive care as prescribed by the surgeon. Patients were followed up until discharge or death. The length of hospital stay (LOS) and mortality as measures of outcome of trauma patients were recorded in days at the end of follow up period. LOS was categorized less than 14 days and more than 14 days as prolonged hospital stay. HIV positive patients were referred to the Care and Treatment Clinic (CTC) after post- counseling.

\section{Data collection}

Data was collected using a pre-tested coded questionnaire. Data administered in the questionnaire included; socio-demographic data; circumstance of injury, characteristics of injury and HIV status and outcome measures.

\section{Data analysis}

Data collected were entered into a computer and analyzed using SPSS software version 11.5. Data were summarized in form of proportions, frequent tables, bar and pie charts for categorical variables. Means, median, mode, standard deviation and histograms were used to summarize continuous variables. Data were categorized into discrete variables and chi-square test was used to test for significance of associations between the predictor and outcome variables in these categorical variables. Hospital stay of more than 14 days was considered as prolonged hospital stay. Odds ratio was calculated to test for strength of association between predictor variables. Significance was defined as a p-value of less than 0.05. Multivariate logistic regression analysis was used to determine predictor variables that are associated with outcome.

\section{Ethical considerations}

The study was carried out after the approval by the 
department of surgery and BMC/WBUCHS Ethics review board. To prevent bias the medical and nursing staff were not informed about the results of the HIV test. All patients were treated according to Bugando Medical Centre protocol which, given the very high prevalence of HIV in general population, assumes that all patients are potentially infected with HIV and staff anticipate HIV related complications and problems in nursing care as a part of daily routine.

\section{Results}

A total of 250 trauma patients were studied. Of these, $202(80.8 \%)$ were males and $48(19.2 \%)$ were females $(\mathrm{M}: \mathrm{F}=4.2: 1)$ with a male predominance in each group. Twenty seven $(10.8 \%)$ patients died and $29(11.6 \%)$ were HIV positive. Sex distribution did not influence the length of hospital stay ( $\mathrm{p}$-value $=$ $0.158)$ and mortality $(P=0.642)$. The age distribution of the study population ranged from 11 to 80 years with the mean of $36.37 \pm 15.35$ years. The peak age incidence was 21-30 years. The age of the patient did not significantly influence the length of hospital stay $(P=0.658)$ and mortality $(P=0.265)$. Nineteen patients $(7.6 \%)$ presented with history of premorbid illness. There was no significant association between pre-morbid illness and either LOS $(P=0.60)$ or mortality $(P=0.23)$. Two hundred and twenty $(88 \%)$ reported to have engaged in risk factors for HIV infection. Of these, alcoholism [Odds Ratio $15.923,95 \%$ C.I. $(2.282-113.240), P=0.001]$ and multiple sexual partners [Odds Ratio 10.208, 95\% C.I. (6.981-14.927), $P=0.000$ ] were found to be independently and significantly associated with increased risk to HIV infection.
Two hundred and thirty five patients (94.0\%) were attended within 24 hours of the injury. The remaining 15 patients $(6.0 \%)$ were attended more than 24 hours since the injury occurred. Timing in seeking medical care did not significantly influence both LOS $(P=0.480)$ and mortality $(P=0.331)$.

A total of 173 injuries $(69.2 \%)$ were unintentional. Intentional injuries occurred in 73 patients (29.2\%) mainly due to physical violence. The remaining four patients $(1.6 \%)$ were cases of undetermined intent. There was no statistically significant association between the nature of injury and either LOS $(P>0.05)$ or mortality $(P>0.05)$. Road traffic accident was the most common cause of injuries affecting 115 (46.0\%). The cause of injury did not significantly influence the outcome of trauma patients in terms of either LOS $(P>0.05)$ or mortality $(P>0.05)$.

Majority of patients $190(76.0 \%)$ sustained blunt injuries. The remaining patients had either penetrating $53(21.2 \%)$ or burn injuries $7(2.8 \%)$. Mechanism of injury did not significantly influence the $\operatorname{LOS}(P>0.05)$ and mortality $(P>0.05)$. Musculoskeletal (extremities) injuries were the most common body region injured affecting 203 patients $(81.2 \%)$. The abdomen was the least affected region $(2.8 \%)$. Body region injured significantly influenced both mortality ( $\mathrm{p}$-value $<0.05$ ) and LOS $(P<0.05)$. Fractures were the most common type of injury affecting 160 patients $(64.0 \%)$. The type of injury; (fractures and burn wounds) significantly influenced the $\operatorname{LOS}(P<0.05)$ but not mortality $(P>0.05)$. Trauma injury scores (ISS, RTS and PTS) significantly influenced LOS $(P<0.05)$ and mortality $(P<0.05)$ (Table 1$)$.

Table 1: Factors associated with prolonged hospital stay (>14days) stay in the study population

\begin{tabular}{|c|c|c|c|c|c|c|c|}
\hline \multirow[t]{2}{*}{ Variable } & \multirow[t]{2}{*}{$\mathbf{N}(\%)$} & \multicolumn{3}{|c|}{ Univariate analysis } & \multicolumn{3}{|c|}{ Multivariate analysis } \\
\hline & & OR & $95 \% \mathrm{CI}$ & $P$ value & OR & $95 \% \mathrm{CI}$ & $P$ value \\
\hline \multicolumn{8}{|c|}{ Injury severity score } \\
\hline $15-49$ & $14(51.8)$ & 1 & $0.61-0.95$ & 0.43 & 1 & & \\
\hline$\geq 50$ & $13(48.2)$ & 5.5 & $0.36-0.78$ & 0.003 & 0.99 & $0.18-0.96$ & 0.0026 \\
\hline \multicolumn{8}{|c|}{ Revised trauma score } \\
\hline$\leq 10$ & $104(44.3)$ & 2 & 1.13 .92 & 0.018 & 2.1 & $1.13-3.92$ & 0.002 \\
\hline$\geq 11$ & $131(55.7)$ & 1 & $0.90-1.27$ & 0.36 & 1 & & \\
\hline \multicolumn{8}{|c|}{ Pediatric trauma score } \\
\hline$\leq 8$ & $2(13.3)$ & 2 & $0.5-7.9$ & 0.002 & 0.08 & $0.003-2.6$ & 0.16 \\
\hline$\geq 9$ & $13(86.7)$ & 1 & $0.79-1.18$ & 0.27 & 1 & & \\
\hline \multicolumn{8}{|c|}{ HIV seropositivity } \\
\hline Yes & $29(11.6)$ & 4.85 & $2.17-10.8$ & 0.0001 & 6.05 & $2.26-16.2$ & 0.0012 \\
\hline No & $221(88.4)$ & 1 & $0.46-0.92$ & 0.625 & 1 & & \\
\hline
\end{tabular}

CD 4+ cell count (HIV positive) 


\begin{tabular}{llllllll}
\hline Variable & $\mathbf{N}(\boldsymbol{\%})$ & \multicolumn{2}{l}{ Univariate analysis } & \multicolumn{3}{c}{ Multivariate analysis } \\
\hline & & OR & $\mathbf{9 5 \%} \mathbf{C I}$ & P value & OR & $\mathbf{9 5 \%}$ CI & P value \\
\hline$<200$ & $8(27.6)$ & 1.83 & $0.35-9.72$ & 0.002 & 0.55 & $0.0010-2.8$ & 0.001 \\
$\geq 200$ & $21(72.4)$ & 1 & $0.1-2.89$ & 0.675 & 1 & & \\
Body region injured & & & & & & \\
Head/Neck & $90(36)$ & 0.83 & $0.44-1.57$ & 0.57 & & & \\
Thoracic & $26(10.4)$ & 0.43 & $0.12-1.49$ & 0.17 & & & \\
Abdomen & $7(2.8)$ & 0.58 & $0.069-4.95$ & 0.62 & & & $0.15-1.11$ \\
Pelvic & $17(7.6)$ & 2.85 & $1.08-7.48$ & 0.028 & 2.4 & 0.080 \\
Extremities & $206(81.2)$ & 0.28 & $0.095-0.81$ & 0.013 & 0.28 & $0.096-0.84$ & 0.023 \\
Multiple & $98(38.2)$ & 0.49 & $0.27-0.89$ & 0.02 & 0.55 & $0.29-1.03$ & 0.06 \\
\hline
\end{tabular}

Of the 250 trauma patients studied, $29(11.6 \%)$ were HIV positive. Of these, 26 patients $(89.7 \%)$ were males and three $(10.3 \%)$ were females. The male to female ratio among HIV positive patients were $8.7: 1$.The peak incidence was in the $31-40$ years age group. There was statistically significant association between HIV seropositivity and either LOS $(P=0.001)$ or mortality $(P=0.001)$. CD4+ count distribution among HIV positive patients ranged from 47 cells $/ \mu 1$ to 570 cells $/ \mu$ with the mean of 246 cells/ $\mu$ l and standard deviation of $99 \mathrm{cells} / \mu \mathrm{l}$. The median and the mode were 230cells/ $\mu \mathrm{l}$ and $150 \mathrm{cells} / \mu \mathrm{l}$ respectively. A total number of eight HIV patients (27.6\%) had CD4+ count below 200 cells $/ \mu$ l and the remaining 21 patients $(72.4 \%)$

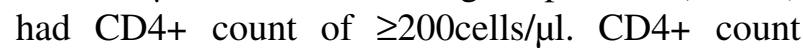
below 200 cells $/ \mathrm{mm}^{3}$ was found to be significantly associated with both $\operatorname{LOS}(P=0.001)$ and mortality $(P=0.001)$. There was no significant difference in the outcome of HIV positive patients with CD4+ count of $>200$ cells / $\mu$ l and HIV negative patients in terms of $\operatorname{LOS}(P=0.675)$ and mortality $(P=0.124)$.

The overall length of hospital stay ranged from 1 to 90 days $($ mean $=19.11 \pm 15.84)$. The median and the mode were 13 days and 9 days, respectively. The LOS for non-survivors ranged from 1 day to 25 days with the mean of $5.60 \pm 7.40$ days. The median was 3 days and the mode was 1 day. According to univariate analysis, the type of injury $(P=0.001)$, ISS $(P=0.003)$, RTS (p-value $=0.018)$, PTS $(P=0.002)$, HIV seropositivity $(P=$ $0.000)$ and CD4+ count $(P=0.002)$ were found to be significantly associated with LOS.

Twenty-seven patients $(10.8 \%)$ in this study died. Of these, 21 patients $(8.4 \%)$ were males and the remaining six $(2.4 \%)$ were females. Mortality rate among patients with moderate injuries (ISS $=16-24)$ was $5.3 \%$. This increased to $78.9 \%$ in patients with severe injuries (ISS $=25-49$ ). Mortality rate among critically injured patients (ISS $=50-75$ ) was $100 \%$. Mortality rates between HIV positive and HIV negative trauma patients were $41.4 \%$ and $6.8 \%$ respectively (Table 2 ). According to univariate analysis, the body region injured $(P<0.05)$, ISS $(P$ $=0.026)$, RTS $(P=0.002)$, PTS $(P=0.01)$, HIV positivity $(P=0.000)$ and CD4+ count $(P=0.035)$ were found to have significant association with mortality.

Table 2: Factors associated with mortality in the study population

\begin{tabular}{|c|c|c|c|c|c|c|c|}
\hline \multirow{2}{*}{ Variable } & \multirow{2}{*}{$\mathbf{N}(\%)$} & \multicolumn{3}{|c|}{ Univariate analysis } & \multicolumn{3}{|c|}{ Multivariate analysis } \\
\hline & & OR & $95 \mathrm{CI}$ & P value & OR & $95 \% \mathrm{CI}$ & P value \\
\hline \multicolumn{8}{|c|}{ Injury severity score } \\
\hline $15-49$ & $14(51.8)$ & 1 & 0.04-3.09 & 0.36 & 1 & & \\
\hline$\geq 50$ & $13(48.2)$ & 1.27 & $1.0-1.59$ & 0.026 & 8.46 & $1.67-42.79$ & 0.002 \\
\hline \multicolumn{8}{|c|}{ Revised trauma score } \\
\hline$\leq 10$ & $104(44.3)$ & 41.14 & $5.47-309.6$ & 0.001 & 41.3 & $5.5-309$ & 0.001 \\
\hline$\geq 11$ & $131(55.7)$ & 1 & $0.68-9.34$ & 0.34 & 1 & & \\
\hline \multicolumn{8}{|c|}{ Pediatric trauma score } \\
\hline$\leq 8$ & $2(13.3)$ & 0.07 & $0.01-047$ & 0.01 & 5.03 & $0.001-101$ & 0.002 \\
\hline$>9$ & $13(86.7)$ & 1 & $0.74-5.96$ & 0.06 & 1 & & \\
\hline \multicolumn{8}{|c|}{ HIV seropositivity } \\
\hline Yes & $29(11.6)$ & 9.74 & $3.94-24.10$ & 0.0001 & 0.04 & $0.01-0.072$ & 0.028 \\
\hline No & $221(88.4)$ & 1 & $0.24-1.39$ & 0.134 & 1 & & \\
\hline \multicolumn{8}{|c|}{ CD 4+ cell count (HIV positive) } \\
\hline$<200$ & $8(27.6)$ & 5.5 & $1.0-28.42$ & 0.035 & 0.3 & $0.055-1.63$ & 0.001 \\
\hline$\geq 200$ & $21(72.4)$ & 1 & $0.35-0.94$ & 0.124 & 1 & & \\
\hline
\end{tabular}




\begin{tabular}{|c|c|c|c|c|c|c|c|}
\hline \multirow{2}{*}{ Variable } & \multirow{2}{*}{$\mathbf{N}(\%)$} & \multicolumn{3}{|c|}{ Univariate analysis } & \multicolumn{3}{|c|}{ Multivariate analysis } \\
\hline & & OR & $95 \mathrm{CI}$ & $P$ value & OR & $95 \% \mathrm{CI}$ & P value \\
\hline \multicolumn{8}{|c|}{ Mechanism of injury } \\
\hline Blunt injury & $190(76)$ & 1 & $0.25-1.92$ & 1 & & & \\
\hline Penetrating injury & $53(21.2)$ & 1.21 & $0.4-3.35$ & 0.72 & & & \\
\hline Burn injuries & $7(2.8)$ & 0.72 & $0.08-6.21$ & 0.76 & & & \\
\hline \multicolumn{8}{|c|}{ Body region injured } \\
\hline Head/Neck & $90(36)$ & 4.2 & $1.81-9.86$ & 0.0001 & \multirow{6}{*}{0.39} & $0.18-0.88$ & 0.019 \\
\hline Thoracic & $26(10.4)$ & 4.82 & $1.85-12.54$ & 0.001 & & & \\
\hline Abdomen & $7(2.8)$ & 6.87 & $1.45-32.56$ & 0.005 & & & \\
\hline Pelvic & $17(7.6)$ & 4.64 & $1.59-13.47$ & 0.002 & & & \\
\hline Extremities & $206(81.2)$ & 1 & $1.25-6.9$ & 0.01 & & & \\
\hline Multiple & $98(38.2)$ & 0.4 & $0.18-0.90$ & 0.02 & & & \\
\hline
\end{tabular}

\section{Discussion}

The prevalence of HIV seropositivity in some trauma population has been reported to be higher than in general population and thus presents an occupational hazard to healthcare workers who care for these patients. The prevalence of HIV infection in the present study was $11.6 \%$ that is higher than that in the general population in Tanzania (6.5\%) (Urassa et al., 2007). This may be attributed to high percentage of the risk factors for HIV infection reported in the present study population. Similar observations have been reported by other studies (Kelen et al., 1989; Caplan et al., 1995; Xeroulis et al., 2005; Grossman \& Stawick et al., 2007). This implies that health care workers who care for trauma patients are at high risk of HIV transmission due to frequent contact with body fluids starting from the Acute and Emergency department to wards and in operating theatres. The overall seroprevalence of HIV in our study was found to be slightly lower than that reported in burn patients in Uganda (13.1\%) (Chalya, 2006). However, a low HIV prevalence in trauma patients of $0.8 \%, 4.3 \%$ and $4.5 \%$ respectively has also been reported in other studies (Behrens et al., 1992; Sloan et al., 1995; Ugwu et al., 2006). This difference in HIV seroprevalence reflects differences in the overall prevalence for risk factors for HIV infection in general population from one country to another.

The age distribution in our study population was similar to other trauma studies in Rwanda, Nigeria and Mozambique (Twagirayezu et al., 2008; Akinpelu et al., 2007; Romao et al., 2003). There was no obvious explanation for the high incidence in this age group.

Our study showed a male preponderance, which is consistent with other trauma studies (Twagirayezu et al., 2008; Romao et al., 2003; Shen-Yong, 2003). Male preponderance was also observed among HIV positive trauma patients. The reason for this observation is probably that males are more mobile with active participation in high risk taking activities.
Majority of patients in this study reported to have risk factors for HIV infection mainly alcoholism and multiple sexual partners. In one study, risk factors for HIV infection were recorded in $21 \%$, and a high prevalence of risk factors for HIV was found in patients with major penetrating wounds (Chambers \& Lord, 2001). In a study in the USA, intravenous drug abuse was found to be the single significant predictor for HIV infection among trauma patients (Sloan et al., 1995). In another trauma study, it was found that patients with positive results for cocaine were more likely than those with negative result to be HIV positive. In that study it was concluded that, young urban trauma patients, because of drug-related intentional violence, were more likely to be infectious for HIV than the trauma population overall (Tardiff et al., 1998).

Majority of injuries were unintentional and occurred following road traffic accidents. Intentional injuries were mainly due to physical violence. Similar results have also been reported in other studies (Twagirayezu et al., 2008; Akinpelu et al., 2007; Romao et al., 2003; Mabrouk et al., 2004). Road traffic accidents are considered to be among the global public health problems (WHO, 2004).

Most patients in this study sustained blunt injuries, which is comparable with other trauma studies (Sodderstrom et al., 1989; Sloan et al., 1995). Musculoskeletal (extremities) injuries were the most common body region injured and were mainly due to fractures. Similar results have been reported in Rwanda and Kenya (Twagirayezu et al., 2008; Otieno et al., 2004). The overall mean LOS in this study was relatively lower than in Nigeria (Akinpelu et al., 2007). Non-survivors in the present study were found to have a shorter mean LOS. A shorter LOS was also reported in the Rwanda study (Twagirayezu et al., 2008). The type of injury, injury scores, HIV seropositivity and CD4+ cell count in our study were all found to be factors significantly associated with LOS. This shows that HIV infection is not the only factor affecting the outcome of trauma 
patients. The present study revealed no significant difference in the time spent as an inpatient between those who were HIV positive with CD4+ cell count 3200 cells/ $\mathrm{ml}$ and those who were HIV negative. Similar findings were also reported by other studies (Chalya, 2006; James et al., 2003; Grossman \& Stawick et al., 2006).

Mortality rates were significantly higher among HIV positive than HIV negative trauma patients in the present study. The mortality rates are comparable to those reported by James et al. (2003) and Chalya (2006). There was no significant difference between HIV positive patients with CD 4 count ${ }^{3} 200$ cells/ $\mu \mathrm{l}$ and HIV negative patients. This observation reflects that the prognosis of HIV positive patients with CD 4 count ${ }^{3} 200$ cells/ $\mu$ is similar to that of HIV negative patients. Our CD4 profile and its influence on the outcome of trauma patients were comparable to that reported in Malawi by James et al. (2003). The body region injured, injury scores, HIV seropositivity and CD4+ count in the present study were found to influence the mortality rate. Despite limited follow-up time and difficult to diagnose HIV infection in its early stage ("window period"), the study has shown that the outcome in trauma patients is dependent upon multiple variables and not the underlying immunodeficiency (i.e. HIV seropositivity and CD4+ cell count) alone. Other factors influencing the outcome of trauma patients include the type of injury, the body region injured and trauma severity scores (ISS, RTS and PTS)

In conclusion, this study has shown that HIV infection is prevalent among trauma patients in Mwanza, Tanzania. A substantial risk of exposure to HIV exists in health care workers who care for these patients. Thus, all trauma health care workers in this region need to practice universal barrier precautions in order to reduce the risk of exposure to HIV infection. HIV positive patients with CD4+ count $\geq 200$ cells/ $\mu$ l have similar prognosis as HIV negative patients and therefore should be treated the same way. The study has also demonstrated that HIV infection is not the only determinant of prognosis in trauma patients; other parameters like the severity and the type of injury should be taken into account. Most of injuries in this study occurred following RTA; therefore, measures on prevention of RTA should be strongly emphasized. Similar study should be conducted in different settings, for a longer duration of time and to include CD4+ count for all patients.

\section{Acknowledgements}

We wish to express our thanks to all the members of staff of the Department of Surgery, Bugando Medical Centre, whose efforts made this study so gratifying. Special thanks go to members of surgical team and A \& E department who were involved in the care of our trauma patients during study period.

Received 28 May 2010

Revised 15 September 2010

Accepted 16 September 2010

\section{References}

Akinpelu, O.V., Oladele, A.O. \& Komolage, A.O (2007) Review of road traffic accident admissions in a Nigerian Tertiary Hospital. East and Central African Journal of Surgery 12, 63-67.

Baraff, L.J., Tallinn, D.A. (1989) Compliance with universal precautions in a university hospital emergency department. Annal Emerging Medicine 18, 654-657.

Behrens, J.J., Stannard, J.P. \& Bucknell, A.L. (1992) The prevalence of seropositivity for human immunodeficiency virus in patients who have severe trauma. Journal of Bone Joint Surgery 74, :641-645.

Caplan, E.S., Preas, M.A. \& Kerns, T. (1995) Seroprevalence of human immunodeficiency virus, hepatitis $B$ virus, hepatitis $C$ virus, and rapid plasma reagin in a trauma population. Journal of Trauma 39, 533-537.

Chalya, P.L. (2006) Factors affecting the outcome of burn injury patients admitted to Mulago Hospital, Kampala. Uganda. Dissertation for Master of Medicine in Surgery. Makerere Medical School, Uganda.

Chambers, A.J. \& Lord, R.S.A. (2001) Documented prevalence of HIV and Hepatitis C infection in patients with penetrating trauma. $A N Z$ Journal of Surgery 1, 21-23.

Courington, K.R., Patterson, S.L. \& Howard, R.J. (1991) Universal precautions are not universally followed. Archive of Surgery 126:93-96.

Dee, J.L. (1990) Injuries in developing countries; emerging health problem. Bulletin of the World Health Organization 77, 518-524.

Hammond, J.S., Eckes, J.M., Gomez, G.A. \& Cunningham, D.N. (1990) HIV, trauma, and infection control: universal precautions are universally ignored. Journal of Trauma 30, 555-558.

James, J., Hofland, H.W., Borgstein, E.S., Kumiponjera, D., Komolafe, O.O. \& 
Zijlstra, E.E. (2003) The prevalence of HIV infection among burn patients in a burn unit in Malawi and its influence on outcome. Burns 29, 55-60.

Kelen, G., Fritz, S., Ququish, B., Floccare, D., DiGiovanna, T., Baker, L., Silvertson, K.T. \& Quinne, T.C. (1989) Substantial increase in HIV-1 in critically ill emergency patients: 1986 and 1987 compared. Annals of Emergency Medicine 18, 378-382.

Lyamuya, E.F., Aboud, S., Urassa, W.K., Sufi, J., Mbwana, J., Ndungulile, F. \& Massambu, C. (2009) Evaluation of rapid HIV assays and development of National Rapid HIV test algorithms in Dar es Salaam, Tanzania. BMC infectious diseases 9:19.

Mabrouk, B., Chelly, H., Ben Hmida, M., Ben Hamida, C., Ksibi, H.,Kallel, H., Chaari, A., Kassis, M., Rekik, N. \& Bouaziz, M. (2004) Prognosis of traumatic head injury in South Tunisia: a multivariate analysis of 437 cases. Journal of Trauma 57, 255-261.

Grossman, M.D. \& Stawicki, S.P. (2006) The impact of human immunodeficiency virus (HIV) on outcome and practice in trauma: Past, present and future. Injury 37, 1117-1124.

Otieno, T., Woodfield, J.C., Bird, P., Hill, A.G. (2004) Trauma in rural Kenya. Injury 35, 1228-1233.

Risi, G., Gaumer, R., Weeks, S., Leete, J. \& Sanders, C. (1989) The risk of exposure to human immunodeficiency virus among health care workers at a southern urban hospital. Southern Medical Journal 82, 1079-1082.

Romao, F. (2003) Road traffic injuries in Mozambique. Injury Control and Safety Promotion10, 63-67.
Sloan, E.P., McGill, B.A., Zalenski, R. (1995) Human immunodeficiency virus and hepatitis B virus seroprevalence in an urban trauma population. Journal of Trauma 38, 736-741.

Soderstrom, C.A., Furth, P.A., Glasser, D.T., Dunning R.W. Groseclose, S.L. \& Cowley, R.A. (1989) HIV infection rates in a trauma centre treating predominantly trauma victims. Journal of Trauma 29, 1526-1530.

Tardiff, K., Marzuk, P.M., Leon, A.C., Hirsch, C.S., Portere, L. \& Hartwell, N. (1998) Human immunodeficiency virus among trauma patients in New York City. Annals of Emergency Medicine 32, 151-154.

Twagirayezu, E., Teteli, R. \& Rugwizangopa, E. (2008) Road traffic injuries at Kigali University Central Teaching Hospital, Rwanda. East and Central African Journal of Surgery 13, 73-76.

Ugwu, B.T., Thacher, T.D., Imade, G.E., Sagay, A.S., Isamade, E.I., Ford, R.W. (2006) HIV and hepatitis B seroprevalence in trauma patients in North Central Nigeria. West Africa Journal of Medicine 25, :6-9.

Urassa, M., Isingo, R., Kumogola, Y., Mwidunda, P., Helelwa, M., Changulucha, J., Mngara. J., Zaba, B., Calleja, T., Slaymaker, E. (2007) Effect of PMTCT availability on choice of ANC in Mwanza and Magu districts and its impact on HIV sentinel surveillance. Report of ANC surveillance Mwanza and Magu Districts, Tanzania.

WHO (1999) A leading cause of Global burden of disease. World Health Organization, Geneva, Switzerland.

WHO (2004) World Report on Traffic Injury Prevention. World Health Organization, Geneva • 